\title{
Changement Climatique et Iniquités en Santé : L'apport de L'approche Interventionnelle en Santé des Populations dans un Contexte Québécois
}

David BUETTI* 1, Rana ANNOUS 2

1 Étudiant(e), Université d'Ottawa, Canada

*Auteur(e) correspondant | Corresponding author : david.buetti@uottawa.ca

Résumé :

Mots-clés :
Dans un monde globalisé, le changement climatique vient s'ajouter à la pléthore de problèmes complexes auxquels la santé publique doit faire face. Cet article propose de s'intéresser, à travers une perspective interventionnelle en santé des populations et dans un contexte québécois, aux conséquences du changement climatique sur les populations vulnérables ainsi qu'aux domaines d'action pouvant les mitiger. Une recherche documentaire a été effectuée dans sept banques de données liées à plusieurs disciplines en sciences sociales, de la santé et de l'environnement. Reconnaissant également l'apport des organismes non gouvernementaux pour la santé des populations, celle-ci a été complémentée par des rapports d'organisations militantes et citoyennes. Les résultats montrent que plusieurs secteurs peuvent collaborer avec les agences de santé publique et les organismes communautaires pour réduire, d'une part, le changement climatique et, de l'autre, les iniquités en matière de santé. Au Québec, trois domaines d'action semblent particulièrement prometteurs, soit : l'aménagement territorial en milieu urbain, le transport et la mobilité durable et l'agriculture urbaine et périurbaine. Préconisant un cadre social écologique et une collaboration multisectorielle, les interventions centrées sur la santé des populations permettent d'atténuer les conséquences du changement climatique sur les iniquités sociales et sanitaires. Quelques défis et pistes de recherche liés à leur implantation et à leur pérennité sont discutés.

Changement climatique, environnements physiques, iniquités en santé, déterminants écologiques de la santé, santé des populations, interventions multisectorielles 


\section{Abstract:}

(translation)

\section{Keywords:}




\section{Introduction}

Dans un monde globalisé, le changement climatique (ciaprès $\mathrm{CC}$ ) vient s'ajouter à la pléthore de problèmes complexes auxquels la santé publique doit faire face. Ses effets sur la santé se manifestent souvent de manière indirecte et touchent les strates de la société de façon inégale (Association canadienne de santé publique [ACSP], 2015). Au Canada, le gouvernement fédéral nouvellement élu en 2015 s'est engagé à agir sur le $\mathrm{CC}$ en réduisant sa progression et en améliorant les capacités des collectivités à le contrer. Les interventions doivent être adaptées au contexte dans lequel elles seront mises en œuvre, en plus de toucher à plusieurs secteurs, comme le transport, l'énergie, l'agriculture, ou l'urbanisme (Watts et al., 2015). Tout comme les autres provinces du Canada, le Québec a des particularités politiques, économiques, sociales, et géographiques qui sont importantes à considérer lors de la conception et la mise en œuvre d'interventions sur le CC (Côté, Logan, et Paquin, 2015; Doyon, Bélanger, et Gosselin, 2008). Pourtant, l'absence de littérature québécoise qui met l'accent sur les iniquités sociales découlant du CC et qui propose conséquemment des pistes d'action intersectorielles et contextuelles pour les mitiger est flagrante. En effet, les études sur le CC restent centrées sur des enjeux populationnels comme la mortalité (Doyon et al., 2008) ou ses conséquences économiques (Bouchard et Smargiassi, 2007) ou psychologiques (Charbonneault et al., 2000), omettant d'identifier, d'une part, les groupes de personnes les plus susceptibles d'en subir les conséquences défavorables en raison d'inégalités sociales et de l'autre, les interventions ayant le potentiel de réduire leurs vulnérabilités et d'améliorer leur résilience, et ce, dans une perspective d'équité et de justice sociale. Pour combler cette double lacune, cet article s'intéresse aux questions suivantes, et ce, dans un contexte québécois :

Quels sont les groupes les plus affectés par les effets du CC sur la santé?

Quels domaines d'actions et pratiques d'interventions ont le potentiel d'atténuer ces conséquences sur la santé de ces populations?

Pour ce faire, nous proposons d'abord une recension des écrits au sujet de la globalisation, du CC et de ses conséquences sur la santé des populations vulnérables. Nous argumenterons ensuite que l'approche axée sur la santé des populations, par ses repères socioécologiques et ses interventions à de multiples niveaux, est en mesure d'atténuer d'une part, le CC et de l'autre, les inégalités sociales de santé. Enfin, pour illustrer son potentiel, nous présenterons trois domaines d'action et de programmes d'intervention prometteurs, qui sont propices au contexte québécois et qui s'appuient sur l'approche axée sur la santé des populations. En conclusion, quelques défis seront explicités quant à la mise en place et à la pérennité de telles interventions.

\section{Matériels et méthodes}

Une recherche documentaire a été effectuée dans sept banques de données liées à diverses disciplines en sciences environnementales, sociales, et de la santé : PsychInfo (psychologie), Sociological Abstract (sociologie), Medline (médecine et épidémiologie), Canadian Public Health Journal (santé publique et des populations), Population

Research and Policy Review (santé des populations et politiques sociales), Érudit (multidisciplinaire - littérature de langue française), et Web of Sciences (multidisciplinaire littérature internationale), en utilisant les mots-clés suivants : " Climate change », "Intervention », « Population Health », «Inequalities », « Québec », et leur équivalent en français. Des rapports gouvernementaux québécois, canadiens, et internationaux ainsi que ceux d'organismes non gouvernementaux ont également été scrutés afin d'en faire émerger les nouvelles pratiques d'intervention, les politiques sociales, et les programmes en voie d'être évalués. Les critères d'inclusion comprenaient la langue (français ou anglais) et la date de publication (à partir de l'année 2000). Pour être qualifiés de prometteurs, les domaines d'action et les programmes d'intervention devaient respecter les postulats des Multiples Interventions Programs qui seront discutés plus spécifiquement dans ce manuscrit, soit : a-toucher au moins un déterminant de la santé; $b$-être actionnable par au moins deux paliers décisionnels, qu'il soit à l'échelle locale, provinciale, ou fédérale; c-utiliser des stratégies multiples à divers niveaux du système écologique; $d$-avoir des retombées positives dans plusieurs domaines sectoriels (ex. environnement et santé); e-être applicables en contexte québécois. Les interventions sélectionnées ont par la suite été présentées à deux professeurs et experts en santé des populations de l'Université d'Ottawa afin d'obtenir leur rétroaction quant à leur pertinence dans le champ interventionnel en santé des populations.

\section{Résultats}

\section{Le CC dans un monde globalisé}

La globalisation, marquée par la planétarisation des échanges et de l'information, a des effets sur les déterminants écologiques de la santé (ACSP, 2015; Buse, 2013; Warren et Lemmen, 2014). L'activité humaine accélérée, 
l'augmentation de la population, l'urbanisation et les normes individualistes qui caractérisent notre époque, affectent plusieurs composantes écologiques nécessaires à la vie en santé comme la qualité de l'air, les ressources alimentaires et hydriques, les cycles de nitrogène et de phosphore nécessaires aux plantes, la fertilité des sols, la douceur des eaux souterraines, les ressources marines et terrestres, une réserve d'énergie suffisante et un climat relativement stable et favorable à la vie (ACSP, 2015; Watts et al., 2015). Les systèmes écologiques sont ainsi menacés, puisque notre empreinte écologique augmente considérablement (dans les pays industrialisés) alors que l'indice planète vivante, une mesure de la biodiversité, diminue sensiblement dans les pays en développement (ACSP, 2015; Watts et al., 2015). Le $\mathrm{CC}$ est une manifestation de ces risques liée notamment à l'augmentation des émissions de gaz à effet de serre. Selon le groupe d'experts intergouvernemental sur l'évolution du climat (GIEC, 2014), le CC persistera pendant plusieurs siècles, même si les émissions de gaz à effet de serre produites par les activités humaines sont réduites, puisque la capacité d'absorption de ces gaz par les surfaces océaniques et terrestres continuera à diminuer.

Les liens entre le CC, la santé, et les inégalités sociales dans le monde et au Québec

Ces changements atmosphériques ne sont pas sans conséquences pour les populations mondiales. En effet, le lien entre le CC et ses répercussions, souvent défavorables pour la santé des collectivités, est bien documenté (Doyon, et al., 2008; Bourque et Willox, 2014; Haines, McMichael, et Epstein, 2000; Last, 2010). Les changements climatiques agissent sur la santé de manière directe, par exemple à travers les catastrophes naturelles ou de manière indirecte à travers les systèmes physiques (glaciers, eau, cours d'eau, mer), les systèmes biologiques (écosystèmes terrestres et marins, incendies de forêt), les systèmes humains (production alimentaire et moyens de subsistance) et les processus sociaux (en exacerbant les migrations, et les conflits en lien avec le manque de ressources; Watts et al., 2015; GIEC, 2014). Parmi les phénomènes découlant du CC les plus préoccupants pour la santé des populations québécoises, on retrouve :

\section{La multiplication des phénomènes météorologi-} ques extremes. Le Québec connaîtra de plus en plus des phénomènes météorologiques imprévisibles (Ministère du développement durable, de l'environnement et de la lutte contre les changements climatiques [DDELCC], 2015; Institut national de santé publique du Québec [INSPQ], 2014; Climat Québec, 2015). En effet, plusieurs acteurs notent l'augmentation importante, sur le territoire québécois, d'épisodes de neige ou de pluie, d'événements météorologiques rares comme les tornades et vents violents, ou d'événements, comme les sècheresses qui perdurent (Côté et al., 2015 ; INSPQ, 2014). Ces phénomènes ont des répercussions directes sur l'environnement physique des résidents et, par conséquent, sur leur santé (Charbonneau et al., 2000; INSPQ, 2014; Warren et Lemmen, 2014). Par exemple, les inondations dérivant d'une accumulation de pluie peuvent amener des effets directs sur la population (p. ex. décès par la noyade; des blessures) ou indirects (p. ex. pertes de revenus; stress; troubles gastro-intestinaux; INSPQ, 2014). Des études indiquent également que ces phénomènes peuvent augmenter la probabilité de souffrir de troubles d'adaptation, notamment ceux liés au stress post-

traumatique (Charbonneau et al., 2000; Warren et Lemmen, 2014). En plus de poser un danger pour la sécurité et le bien-être de la population, la multiplication de ces phénomènes aura d'importantes répercussions sur l'économie. L'une des plus grandes crises ayant touché le Québec, celle du Verglas en 1996, a causé plus de six milliards de dollars en pertes économiques, en plus nombreuses pertes humaines.

Certes, la capacité de fuir les intempéries ou de se protéger ne semble pas égale à travers la société. Elle est liée aux contextes sociaux dans lesquels vivent les individus et les groupes (Maltais et Gauthier, 2008). Bien que les données québécoises soient plus rares à ce sujet, une pluralité de rapports officiels (GIEC, 2014; Victorian Council of Social Science [VCOSS], 2014) et d'études internationales (Hoffman, 2009; Watts et al., 2015) explique que le statut socioéconomique d'une personne peut grandement l'influencer dans sa capacité à réagir aux événements météorologiques. Des 173 personnes décédées en 2009 après un feu de forêt en Australie, plus de la moitié était des personnes âgées ayant peu de ressources sociales et financières pour être évacués rapidement (VCOSS, 2014). Des 1800 personnes décédées par les inondations suivant l'ouragan Katrina, Hoffman (2009) rapporte une disproportion significative de personnes de race noire, de personnes ayant un handicap et de personnes âgées dont le statut socioéconomique était faible. En clair, si le CC peut affecter la santé de l'ensemble des Québécois par la multiplication de phénomènes météorologiques extrêmes, il est possible de croire que ces derniers toucheront plus fortement les personnes désavantagées sur le plan socioéconomique et souffrant d'exclusion sociale en raison, par exemple, de leur âge, d'une situation d'handicap ou de leur appartenance à une minorité visible.

\section{Hausse de la température et des vagues de chaleur.}

Le réchauffement climatique provoquera une augmentation significative des vagues de chaleur au Québec. Une vague de chaleur peut se comprendre comme étant une température 
au-dessus de $30^{\circ} \mathrm{C}$ accompagnée d'un indice d'humidité de plus $40^{\circ} \mathrm{C}$, et ce, pour au moins deux journées consécutives (INSPQ, 2010a). Santé Canada (2012) estime que le nombre de ces journées doublera fort probablement dans les cinquante prochaines années dans plusieurs villes canadiennes et de la province de Québec. Les vagues de chaleur peuvent avoir des effets directs sur la santé des populations. En effet, la chaleur accablante peut mener les individus à souffrir des maux de tête, des périodes d'épuisement, de déshydratation et de crampes musculaires (Barna, Goodman, et Mortimer, 2012; Gower, 2011). Dans des cas extrêmes, les vagues de chaleur peuvent provoquer des pertes de conscience, voire même la mort (Gower, 2011; Sheffield et Landrigan, 2011). Après avoir examiné l'effet possible du CC sur le taux de mortalité dans trois villes québécoises (Montréal, Québec, et Saguenay), Doyon et ses collègues (2008) ont projeté que le CC allait contribuer significativement à l'augmentation des décès en été dans les prochaines années. D'ailleurs, deux ans après la publication de leurs résultats, au mois de juillet 2010, le Québec a vécu l'une de ses pires canicules. L'analyse qui en a été faite par Bustinza et ses collègues (2013) révèle qu'environ 280 Québécois en sont décédés, soit environ $33 \%$ de plus en comparaison aux semaines équivalentes des années précédentes. Parallèlement, la vague de chaleur a contribué à plus de 3400 hospitalisations, provoquant un important achalandage dans les urgences de la province (Bustinza et al., 2013).

Les individus qui résident en ville et qui ont un faible statut socioéconomique sont particulièrement à risque de subir les contrecoups des vagues de chaleur (Laaidi, 2012; Watts et al., 2015). En effet, ils sont plus susceptibles de vivre, grandir et s'amuser à l'intérieur « d'îlots de chaleurs ». Produits de la déforestation urbaine, de l'étalement des villes et du transport, les îlots de chaleur peuvent augmenter la température ambiante de $12^{\circ} \mathrm{C}$ en milieu urbain en comparaison au milieu rural (Giguère, 2009; Laaidi, 2012). Les personnes défavorisées en milieu urbain sont plus à risque de souffrir des symptômes liés à la chaleur accablante puisqu'ils sont plus enclins à habiter dans des logements non climatisés (p. ex. des tours en hauteur) et ont moins accès aux transports ou aux congés permettant de quitter les zones de chaleur (INSPQ, 2010b; Keller, 2013; Watts et al., 2015). Keller (2013) déclare que le risque de mourir durant les vagues de chaleur est un problème davantage social que biologique. Ses observations concordent avec celles de Klinenberg (1999) qui avait remarqué que la majorité des décès suivant la vague de chaleur à Chicago, en 1995, se produisaient à l'intérieur de quartiers où la pauvreté se conjuguait à des infrastructures comme des logements de piètre qualité ou des espaces verts réduits. L'intersection entre le fait de vivre dans la pauvreté et d'avoir un âge élevé est également un facteur de risque important. Des 15000 décès occasionnés par la vague de chaleur en 2003 sur le territoire français, environ $80 \%$ avaient plus de 75 ans, souvent en situation de pauvreté et d'isolement social (Gower, 2011; Sheffield et Landrigan, 2011).

Parallèlement, la hausse des températures et les chaleurs accablantes peuvent amener des effets indirects sur la sécurité alimentaire (Watts et al., 2015). Le CC contribue à la diminution de la qualité des récoltes pouvant mener à une augmentation globale des prix de la nourriture, et ce, même au Canada (Lewis et Witham, 2012; Watts et al., 2015). Le prix élevé de la nourriture peut, à son tour, forcer les individus vivant dans une situation de précarité à consommer des aliments de piètre qualité, augmentant ainsi les risques de maladies chroniques (ACSP, 2015). Les résultats de l'enquête Bilan-Faim rapportent d'ailleurs une forte augmentation entre 2008 et 2011 (22\%) des demandes d'aide alimentaire au Québec (Pegg, 2011). Bien que d'autres facteurs que le CC puissent expliquer cette augmentation, l'Institut national de santé publique du Québec (2010c) affirme que le CC fait et continuera de faire partie des responsables de l’insécurité alimentaire chez les résidents de la province.

Pollution atmosphérique et santé. La hausse des températures qui est principalement anthropogénique (c.-àd. occasionnée par les activités humaines) favorisera, non seulement la fréquence et la sévérité des canicules, mais aussi la formation de pollution atmosphérique (Bouchard et Smargiassi, 2007; Environnement Canada, 2013a). Le rapport Bouchard et Smargiassi de la Direction des risques biologiques, environnementaux et occupationnels, révèle qu'en 2002 seulement, le Québec a perdu près de 2000 personnes en raison des particules fines se trouvant dans la pollution atmosphérique. Ce nombre élevé de décès s'accompagne d'au moins 500 visites aux urgences, principalement liées aux problèmes cardiaques et respiratoires en plus d'occasionner des coûts importants frôlant les dix milliards de dollars (Bouchard et Smargiassi, 2007).

Encore une fois, les études montrent que la pollution touche particulièrement les individus résidant en ville, surtout ceux ayant un faible statut socioéconomique (Finkelstein et al., 2003; Neidell, 2004; Wong et al., 2008). Dans le contexte canadien, une étude de l'effet de la pollution atmosphérique sur le taux de mortalité de 5228 personnes à Hamilton montre que les personnes défavorisées étaient plus nombreuses à mourir des suites de maladies cardio-pulmonaires (Finkelstein et al., 2003).

En somme, le portrait présenté ci-dessus démontre au moins deux constats : 1) le CC a des effets directs et indirects sur la santé des collectivités. Ces effets s'accentueront au cours des prochaines années; 2) Les populations sont 
touchées de façon inégale par le CC. L’intersection entre les facteurs biologiques (l'âge) et socioéconomiques (pauvreté, insécurité alimentaire, et de logement) ou géographiques (milieu urbain) a été retenue comme un facteur de risque important. En effet, les Québécois aux extrêmes d'âge (enfants et personnes âgées), qui sont désavantagés socioéconomiquement et qui vivent en milieu urbain sont ainsi plus vulnérables quant à la multiplication des phénomènes météorologiques extrêmes, aux vagues de chaleur, à l'insécurité alimentaire, ou à la pollution atmosphérique. Ces conclusions guideront le reste de ce manuscrit, notamment dans la discussion de l'approche à adopter et les interventions à mettre en place.

\section{Discussion}

Les résultats présentés n'ont rien de surprenant. Les travaux en épidémiologie et en médecine sociale ont depuis longtemps indiqué la présence d'un gradient social en santé, soit une corrélation positive entre l'état de santé et le positionnement qu'un individu occupe dans la hiérarchie sociale (voir les travaux de Krieger, 2012; Navarro, 2002; Wilkinson, 2010). Ce gradient est le résultat d'inégalités structurelles dans les opportunités de s'actualiser sur le plan de la santé. Elles résultent tant de l'organisation de la société que de ses choix politiques, économiques et sociaux. Ainsi, prétendre qu'une seule caractéristique (l'âge chez les personnes âgées, par exemple) explique qu'ils aient plus de difficultés à s'adapter au CC serait, non seulement erroné, mais pourrait minimiser l'effet des autres facteurs dans l'entretien de cette dynamique.

On reconnaît maintenant l'importance d'agir sur les iniquités en matière de santé, notamment par l'approche axée sur la santé des populations (ASPC, 2012). Contrairement aux paradigmes interventionnels mettant principalement l'accent sur les facteurs individuels des maladies (c.-à-d. biomédical), l'approche axée sur la santé des populations utilise un cadre holistique lui permettant de repérer l'interconnexion de diverses influences (p. ex. psychologique, socioculturelle, politique) sur la santé d'une population. Elle concède ainsi que le bien-être collectif ne peut être attribuable uniquement à l'efficacité de son système de soins et qu'elle se doit, dans une perspective d'équité et de justice sociale, d'attaquer les inégalités en matière de santé (ASPC, 2012; Evans et Stoddart, 1990).

Pour Edwards et ses collègues (2004; 2006), explorer les interconnexions entre les dimensions individuelles et collectives ainsi que leurs boucles de rétroactions (c.-à-d. comment les systèmes se modifient et s'interinfluencent) permet d'éviter « le blâme des victimes » ou, autrement dit, d'éviter de responsabiliser les individus qui ne disposent pas des ressources nécessaires pour s'adapter adéquatement au CC. Pour ce faire, l'auteure suggère l'adoption d'un modèle socioécologique axée sur la santé des populations (Edwards, 1999; Edwards et Di Ruggiero, 2011). Ces modèles ont pour particularité d'intégrer les déterminants sociaux de la santé dans l'analyse d'une problématique. Plus précisément, ils réitèrent que cette dernière a bien souvent des causes qui dépassent le spectre individuel. Par conséquent, afin d'améliorer la santé d'une collectivité, il est essentiel d'opter aussi pour des stratégies en amont, c'est-à-dire qui améliorent l'environnement dans lequel elle évolue (Edwards et al., 2004), tout en considérant son contexte social, historique et politique (Edwards et Di Ruggiero, 2011). Une approche axée sur la santé des populations doit miser sur des stratégies et des interventions multiples, aussi appelées les Multiple Interventions Program (ci-après MIPs). Les MIPs se caractérisent par l'utilisation simultanée de plusieurs stratégies (c.-àd. politiques sociales, interventions communautaires, soins directs), ciblées à divers niveaux du système socioécologique (c.-à-d. contexte global, systèmes, milieux, individus) et parfois livrées à des populations spécifiques (p. ex. les individus ayant un faible statut socioéconomique). Considérant le caractère multidimensionnel des MIPs, l'action intersectorielle devient non seulement souhaitable, mais nécessaire dans les interventions subséquentes.

L'intersectorialité peut se comprendre comme une préoccupation partagée par plusieurs paliers (c.-à-d. palier fédéral, provincial, municipal) et ministères (p. ex. ministre de l'Agriculture, de l'Environnement) afin d'adresser, de façon concertée, une problématique (Edwards, 1999; Edwards et al., 2006). Pour illustrer le potentiel des MIPs sur les iniquités de santé exacerbées par le CC, trois domaines d'action différents, mais complémentaires sont présentés. Elles sont tirées du contexte québécois et se basent sur les vulnérabilités identifiées plus haut. En effet, nous montrerons comment des investissements dans des secteurs intersectoriels touchants : a) l'aménagement du territoire urbain, b) le transport et la mobilité durable et c) l'agriculture urbaine et périurbaine, peuvent avoir des retombées positives sur plusieurs déterminants sociaux et écologiques de la santé.

\section{Aménagement du territoire urbain}

L'aménagement du territoire est tributaire d'une bonne santé sur les plans collectifs et individuels. Les îlots de chaleur en milieu urbain contribuent aux problèmes de santé, notamment lors de vague de chaleur accablante. Sans l'implantation de mesures visant adapter le territoire québécois, les victimes de vague de chaleur et des hausses 
de températures seront accrues au cours des prochaines années (Doyon et al., 2008). Les personnes qui habitent dans un quartier défavorisé sont particulièrement vulnérables aux îlots de chaleurs, n'ayant pas aussi facilement accès à des infrastructures de qualité (p. ex. parcs, logements climatisés, etc.).

Les recherches suggèrent fortement de lutter contre les îlots de chaleurs urbaines, notamment par l'augmentation de l'indice de végétalisation des municipalités (Giguère, 2009; Heinze, 2011; Watts et al., 2015). Pour ce faire, au moins deux types de stratégies complémentaires sont nécessaires : a) la bonification des espaces verts en ville et b) l'amélioration durable des infrastructures. Concrètement, ces stratégies se traduisent par des mesures de plantation ponctuelle d'arbres dans les quartiers et les stationnements, la construction de toits verts et de murs végétaux, la construction de parcs et de platebandes dans les ruelles (Giguère, 2009). Le projet ILEAU de Montréal (Interventions locales en environnement et aménagement urbain) est ici présenté comme une intervention prometteuse qui propose d'attaquer les îlots de chaleurs par une stratégie à multiples niveaux systémiques (ILEAU, 2015). Au niveau communautaire, le programme propose le verdissement des toits, la transformation des stationnements en espaces verts, et la construction de parcs sécuritaires et conviviaux pour les citadins (Communauté métropolitaine de Montréal [CMC], 2015). Pour engager et mobiliser la communauté, le projet ILEAU-Montréal offre des stratégies telles que le porte-àporte (niveau interindividuel), des rencontres dans les écoles primaires et secondaires (niveau intracommunautaire), des partenariats avec des organismes communautaires, et la création de comités de mobilisation et d'action citoyenne (p. ex. planter des arbres). Le projet ILEAU touche ainsi à une diversité de citoyens et s'avère, dans la pratique, un projet prometteur puisqu'il semble s'appuyer sur les postulats de l'approche de la mobilisation communautaire. Cette dernière se démarque par ses actions et ses analyses, reconnaissant aux facteurs environnementaux un rôle déterminant dans la santé physique et mentale des sociétés (Doucet et Favreau, 1991). C'est par la collectivisation des problématiques, soit une réflexion sociocritique sur son quartier et ses défis, que s'enclenchera un désir d'engagement et de changement durable. Par conséquent, l'implication citoyenne est cruciale dans l'intervention puisqu'elle reconnait que les « experts » sont ceux qui vivent de façon continue dans les quartiers : " (le projet est) mené en étroite collaboration avec les partenaires de mobilisation locaux afin d'assurer l'engagement du plus grand nombre d'organisations et ainsi créer un effet d'entraînement » (INSPQ, 2015, p.2).
Plusieurs études soutiennent que ce type de projet a des retombées positives et durables pour la santé des populations et pour l'environnement. Parmi les rapports analysés, notons l'amélioration de la qualité de l'air du quartier (Amorim, Rodrigues, Tavares, Valente, et Borrego, 2013; Giguère, 2009; Zupancic, Bulthuis, et Westmacott, 2015), la réduction de sa pollution atmosphérique (Heinze, 2011;

Zupancic et al., 2015), la diminution de la température ambiante et au sol (Giguère, 2009; Zupancic et al., 2015) ainsi que la réduction des coûts liés à la climatisation (Heinze, 2011). On note également qu'elle promeut et facilite l'activité physique et le bien-être, et favorise les contacts sociaux dans les collectivités (Alcock, White, Wheeler, Fleming, et Depledge, 2014; Heinze, 2011). Une caractéristique particulièrement notable de l'intervention est sa sensibilité aux inégalités sociales. Comme il a été mis en relief auparavant, les îlots de chaleurs sont très présents dans les quartiers défavorisés. Par conséquent, le projet ILEAU s'implante principalement dans les quartiers ayant une plus grande concentration de personnes à faible statut socioéconomique (ceux de l'Est de Montréal). Ce paramètre est particulièrement important pour assurer, d'une part, la santé des collectivités, mais également la réduction des iniquités de santé chez les plus vulnérables.

\section{Transport et mobilité durable}

Le transport demeure l'un des secteurs qui contribue le plus à la pollution atmosphérique dans la province de Québec (Fondation David Suzuki, 2014; Union des municipalités du Québec [UMQ], 2008). Cette dernière occasionne de nombreux problèmes de santé en milieu urbain où un peu plus de 80\% de la population québécoise réside (Institut de la statistique du Québec [ISQ], 2015). Les quartiers défavorisés sont durement touchés par la pollution atmosphérique : ils seront plus nombreux à en souffrir et en mourir (Bouchard et Smargiassi, 2007). Pour contrer ces effets néfastes sur la santé, les études suggèrent fortement de développer une offre plus active et attrayante de transports durables (Barna et al., 2012; Litman, 2015; Transport Canada [TC], 2009). Concrètement, au plan ministériel, cette offre doit se traduire par une politique visant à soutenir les municipalités dans cet effort. À court terme, l'introduction des politiques « Complete Streets » (ci-après $\mathrm{CS}$ ) mènera vers le réaménagement d'axes routiers plus verts et durables. À long terme, un système efficace de transport actif et collectif permettra non seulement de diminuer la pollution atmosphérique (p. ex. moins de congestions), mais de promouvoir un mode de vie actif au sein de la population (p. ex. augmentation des cyclistes dans une municipalité).

Le CS est une intervention en pleine effervescence aux États -Unis (Seskin et Gordon-Koven, 2013) et qui prend beau- 
coup d'ampleur en Ontario où elle est financée en partie par le gouvernement provincial (Toronto Centre for Active Transportation [TCAT], 2015). L'intervention vise à planifier, à aménager et à repenser nos routes afin qu'elles puissent accueillir, de façon sécuritaire et attrayante, une diversité de modes de transports (c.-à-d. le vélo, la marche et le transport en commun) et d'utilisateurs (p. ex. personne âgée, les jeunes, les mères monoparentales). La voiture devient ainsi un moyen parmi tant d'autres de se déplacer en ville, les autres étant devenus tout aussi agréables et efficaces (Litman, 2015).

Le CS s'inscrit dans la visée des interventions MIP pour plusieurs motifs. D'abord, il se formalise à divers niveaux par un engagement politique des municipalités et de la province au profit de routes qui sont conçues pour être accessibles pour tous. Ensuite, il utilise de multiples stratégies pour en arriver à repenser l'aménagement des routes. Sur le plan de l'urbanisme, l'élargissement des trottoirs, l'augmentation de la fréquence des autobus, la réduction de la vitesse permise pour les voitures, la construction de sentiers pédestres et cyclables et de nouvelles voies réservées aux autobus et covoitureurs figurent parmi les stratégies les plus courantes (Litman, 2015). Les stratégies d'urbanisme sont également accompagnées de stratégies communautaires. Par exemple, des comités citoyens dont la mission est de promouvoir et d'assurer les besoins découlant des gens du quartier sont mis en place (Litman, 2015; TC, 2009). La configuration des aménagements répond alors aux besoins d'une diversité de personnes comme les personnes âgées, les enfants ou les personnes à mobilité réduite. Le CS est aussi sensible au contexte de chaque municipalité. En effet, l'adoption des politiques $\mathrm{CS}$ ne mène pas nécessairement à une reconfiguration complète des routes construites. Plutôt, la municipalité, en étroite concertation avec le gouvernement et les citoyens, détermine les zones à prioriser en se basant sur plusieurs indications : nombre d'accidents, temps de congestion, nombre de cyclistes, etc. (Smart Growth America, 2015). À cet effet, un guide d'implantation complet permet d'adapter le CS aux réalités vécues par une communauté (voir le site Internet de Smart Growth America, 2015).

De nombreuses recherches ont évalué les retombées des CS. Ces dernières ont relevé des répercussions favorables sur l'environnement, la santé et même sur l'économie (Litman, 2015; New York City Department of Transportation [NYCDOT], 2012; TC, 2009). Plus spécifiquement, le rapport du ministère du Transport de New York (NYCDOT, 2012, p. 22-41) montre qu'après l'implantation de CS, le nombre de piétons et de cyclistes blessés par les automobiles avait diminué entre $7 \%$ et $56 \%$ sur certaines artères de Manhattan. Parallèlement, la ville a assisté à une augmentation d'au moins 10\% des usagers de transports en commun, à une réduction de la congestion routière d'au moins $7 \%$ et à une réduction des émissions de gaz à effet de serre de $9 \%$ aux heures de pointe. Ces données sont assez similaires à celles de Portland où les politiques CS ont mené à une "réduction de 12,5\% des émissions de dioxyde de carbone liées au transport » (TC, 2009, p. 2 - traduction libre). Qui plus est, puisque les artères deviennent plus attrayantes, le CS a stimulé l'économie de plusieurs quartiers new-yorkais en diminuant de près de moitié (47\%) le taux d'inoccupation commerciale dans les rues visées par le programme (NYCDOT, 2012, p. 9). Un rapport canadien a lui aussi dressé un portrait positif de l'intervention, suite à son implantation dans la ville de Victoria. Les auteurs notent une « amélioration de l'accessibilité du territoire pour les personnes sans automobiles, des économies sur l'entretien des routes et des stationnements, l'amélioration de la condition physique des résidents et de la santé publique, la conservation de l'énergie, de même que la diminution de la pollution sonore et des gaz à effet de serre» (Litman, 2015, p. 22 traduction libre).

\section{Agriculture urbaine et périurbaine}

Tel que discuté plus haut, la hausse des températures contribuera à la multiplication des phénomènes météorologiques extrêmes qui, à leur tour, peuvent mener vers d'importantes pertes agricoles au Canada comme à l'international. Ces pertes ont des répercussions directes sur la disponibilité de produits locaux à des prix raisonnables. En l'absence d'une sécurité alimentaire stable, les populations vulnérables peuvent avoir de la difficulté à s'alimenter convenablement, menant ainsi à divers troubles de santé (ex. maladies chroniques, obésité). Le rapport Béliveau (2007) présenté à la commission sur l'avenir de l'agriculture et de l'agroalimentaire du Québec se veut assez transparent sur le modèle d'agriculture qui prévaut dans la province : « $\mathrm{Au}$ Québec, l'agriculture dans les régions rurales ne permet plus de subvenir aux besoins des populations urbaines sur le plan de la diversité alimentaire. Les habitants des villes n'ont d'autre choix que d'aller vers le marché de l'alimentation, qui offre des fruits et légumes transportés sur de grandes distances, ce qui coûte cher, pollue et réduit la qualité des aliments » (p. 4). À ces problèmes, s'ajoutent ceux déjà occasionnés par les îlots de chaleur en milieu urbain (Giguère, 2009) et ceux liés à l'augmentation constante du coût de la vie (Lewis et Witham, 2012).

L'agriculture urbaine et périurbaine (ci-après AUP) apparait dans la littérature comme une intervention durable et écologique freinant l'insécurité alimentaire tout en agissant à long terme sur le CC (Alaimo, Packnett, Miles, et Kruger, 2008; Béliveau et al., 2007; Suarez-Balcazar, Martinez, Jayraj, et Cox, 2006). L'AUP peut se comprendre comme les 
formes d'activités qui visent à produire, à manufacturer et mettre sur le marché des aliments variés pour la population en milieu urbain ou périurbain (c.-à-d. en bordure des banlieues). Elle utilise de multiples stratégies tels les jardins communautaires (stratégie communautaire), les marchés locaux (stratégie municipale) et les politiques agricoles (stratégie provinciale) à plusieurs niveaux (agriculteurs et bénévoles, organismes communautaires, municipalités, gouvernement) pour arriver à ses fins. Certes, réduire l'AUP à sa composante de production serait d'ignorer ses nombreux bénéfices au plan environnemental, social, et de la santé. L'étude de Alaimo et ses collègues (2008) montre que les personnes défavorisées qui participent aux jardins communautaires sont près de 3.5 fois plus susceptibles de manger des légumes et des fruits cinq fois par jour ( $\mathrm{n}=$ 766). Celle de Suarez-Balcazar et ses collaborateurs (2006) soulèvent que parmi une communauté afro-américaine, les marchés locaux découlant des AUP mènent à un taux très élevé de satisfaction quant à la qualité et les coûts des produits, menant la communauté à en consommer davantage. La revue de littérature de Golden (2013) soutient à partir de données internationales que l'AUP peut également promouvoir le sentiment d'appartenance à la communauté de même qu'à la création d'emplois pour les plus démunis. Les rapports Béliveau (2007) et Boily (2012) relèvent également ces effets positifs sur l'environnement urbain et périurbain. Ceci est notamment lié au fait que l'AUP diminue le transport associé à l'importation d'aliments et permet de créer de nouveaux espaces verts.

L'un des défis majeurs de l'AUP réside cependant dans sa principale force : elle est intersectorielle. En effet, puisque ses retombées touchent plusieurs domaines (santé, agriculture, environnement), elle trouve du mal à se faire financer de façon durable par un bailleur de fonds. Ainsi, les organismes communautaires peuvent être subventionnés pour certains de ses volets (p. ex. le volet promotion des saines habitudes de vie ou celui de la lutte aux îlots de chaleurs), sans toutefois qu'elle soit considérée comme une intervention complète en soi. Au Québec, plusieurs déplorent que le ministre de l'Agriculture n'ait pas encore reconnu formellement l'agriculture urbaine comme une forme durable d'agriculture, contribuant par le fait même à son sousfinancement et à son statut marginal en société (Béliveau et al., 2007). Par conséquent, l'AUP peut être perçue comme une menace par les projets privés de développements urbains et de banlieue (Boily, 2012).

\section{Conclusion}

Le CC aura des conséquences profondes sur les collectivités et leurs populations vulnérables, au Québec comme ailleurs.
Pour les contrer, une volonté politique (Watts et al., 2015), intersectorielle (Buse, 2013) et systématiquement mue par des valeurs d'équité (Walpole, Rasanathan, et CampbellLendrum, 2009) est nécessaire. Un récent rapport de l'Institut de médecine (2015) avance que la « la santé, l'équité, la durabilité et la résilience font partie du même cercle vertueux ». Dans ce sens, l'approche centrée sur la santé des populations s'aligne avec les principes de la promotion de la santé et des déterminants sociaux de la santé présentés par la déclaration d'Alma Ata, la Charte d'Ottawa et la Commission sur les déterminants de la santé de l'OMS (Health Resources in Action, 2013; Patrick, Capetola, Townsend, et Nuttman, 2012; Patrick, Noy, et Henderson-Wilson, 2015). Elle réitère que le bien-être d'une société dépasse largement l'accessibilité aux services de santé. En effet, des interventions, qui à priori ne sont pas ciblées pour la santé, peuvent avoir des répercussions positives tant sur le plan de l'environnement que sur les iniquités en matière de santé. $\mathrm{Au}$ Québec, des actions multiples dans les secteurs de l'aménagement urbain, du transport et de l'agriculture offrent des avenues prometteuses permettant d'atténuer les émissions anthropogéniques de gaz à effet de serre, d'améliorer la résilience face aux adversités climatiques et de réduire les iniquités sociales en santé.

Force est d'admettre que la compartimentation des ministères, ayant chacun leur propre culture et leurs propres objectifs à atteindre, complexifie grandement la mise en place de telles collaborations (Greaves et Bialystok, 2011). Le secteur de la santé, en collaboration interdisciplinaire avec d'autres champs, a donc un rôle majeur à jouer. Le discours sur le CC doit être encouragé par les leaders en santé (professionnels et administrateurs), notamment en avançant les cobénéfices pour la santé de la mitigation du changement climatique, d'autant plus que plusieurs maladies, notamment celles chroniques, partagent des voies causales similaires à celle du CC (Smith et al., 2014; Watts et al., 2015) et profitent simultanément des interventions comme l'agriculture urbaine ou une diminution de l'utilisation des transports motorisés. Qui plus est, afin d'optimiser leur durabilité, les interventions nécessitent une collaboration avec la communauté favorisant ainsi l'autonomisation. Sans une implication de la communauté, certains de ces projets peuvent vite tomber sous le radar de l'embourgeoisement ou seront voués à l'échec en raison de leur inadéquation contextuelle. Dans ce sens, il est pertinent de considérer les villes comme unité de changement et de résilience (Bentley, 2007; Hancock, 2009; Health Resources in Action, 2013; Institute of Medicine, 2015; Jabareen, 2013; Watts et al., 2015), tel qu'il a été proposé dans ce travail. Les villes, à travers leur monde, ont entrepris des actions pour faire face au CC, implantées par leurs municipalités de manière plus flexible qu'à l'échelle nationale. Cela a constitué une sorte 
de « gouvernance par le milieu » qui, de manière transversale, a lié plusieurs villes à travers le monde (Watts et al., 2015). La gouvernance au niveau local permet d'adopter la vision « Santé dans toutes les décisions » de manière plus souple en surmontant les conflits liés aux idéologies politiques à l'échelle nationale. De plus, les actions locales permettent d'évaluer la mise en œuvre des interventions dans des contextes restreints et de comprendre les besoins, les croyances et les pratiques avant de planifier le changement.

Des recherches plus approfondies sont nécessaires pour appuyer ces arguments. Un constat est néanmoins apparent : la réussite des activités proposées nécessite un effort descendant des politiques gouvernementales, et un effort ascendant de l'engagement citoyen, afin de pouvoir « craquer la noix de l'équité » (Baum, 2007) et de moduler les conditions écologiques et sociales affectant la santé planétaire et humaine.

\section{Remerciements}

Nous remercions chaleureusement Julien ThibaultLévesque, étudiant au doctorat en service social (Université d'Ottawa), ainsi que les deux réviseurs de la Revue Interdisciplinaire des Sciences de la Santé pour leurs commentaires constructifs sur la version initiale et révisée de ce manuscrit. Nous souhaitons également remercier le Dr Sanni Yaya (Université d'Ottawa) et la Dre Caroline Andrews (Université d'Ottawa) pour leurs réflexions entourant les interventions choisies dans cet article.

\section{Note}

${ }^{1}$ Les praticiens en santé publique et des populations qui s'intéressent à cette méthodologie d'intervention peuvent trouver un coffre à outils complet à l'adresse suivante : http://www.miptoolkit.com (en anglais seulement).

\section{Références}

Agence de la santé publique du Canada. (2012). Qu'est-ce que l'approche axée sur la santé de la population? Repéré à http://www.phac-aspc.gc.ca/ph-sp/approach-approche/ index-fra.php

Alaimo, K., Packnett, E., Miles, R. A., \& Kruger, D. J. (2008). Fruit and vegetable intake among urban community gardeners. Journal of Nutrition Education and Behavior, 4O(2), 94-101. doi:10.1016/j.jneb.2006.12.003
Amorim, J. H., Rodrigues, V., Tavares, R., Valente, J., \& Borrego, C. (2013). CFD modelling of the aerodynamic effect of trees on urban air pollution dispersion. Science of the Total Environment, 461, 541-551. doi:10.1016/

j.scitotenv.2013.05.031

Association canadienne de santé publique. (2015). Les changements globaux et la santé publique : Qu'en est-il des déterminants écologiques de la santé? Repéré à http:// www.cpha.ca/uploads/policy/edh-discussion_f.pdf

Barna, S., Goodman, B., \& Mortimer, F. (2012). The health effects of climate change: What does a nurse need to know? Nurse Education Today, 32(7), 765-771. doi:10.1016/ j.nedt.2012.05.012

Baum F. (2007). Cracking the nut of health equity: Top down and bottom up pressure for action on the social determinants of health. Promotion \& Education, 14(2), 90-95. doi:10.1177/10253823070140022002

Béliveau, A., McMeekin, K., Lafleur, C., \& Trottier, A. (2007). Le rôle de l'agriculture urbaine dans le développement de la société québécoise. Repéré à http://

www.caaaq.gouv.qc.ca/userfiles/File/Memoires\%

20Gatineau/o7-28-GForum_institut_sciences_environnement.pdf

Bentley, M. (2007). Healthy cities, local environmental action and climate change. Health Promotion International 22(3), 246-53. doi:10.1093/heapro/damo13

Boily, M. É. (2012). L'agriculture périurbaine et urbaine au Québec : État de situation et perspectives. Repéré à http:// www.mapaq.gouv.qc.ca/fr/Publications/Agricultureurbaine etperiurbaine.pdf

Bouchard, M., \& Smargiassi, A. (2007). Estimation des impacts sanitaires de la pollution atmosphérique au québec : Essai d'utilisation du air quality benefits assessment tool (aqbat). Repéré à https://www.inspq.qc.ca/pdf/ publications/817_ImpactsSanitairesPollutionAtmos.pdf

Bourque, F., \& Willox, A. C. (2014). Climate change: The next challenge for public mental health? International Review of Psychiatry, 26(4), 415-422.

doi:10.3109/09540261.2014.925851

Buse, C. (2013). Intersectoral action for health equity as it relates to climate change in Canada: Contributions from critical systems heuristics. Journal of Evaluation in Clinical Practice, 19(6), 1095-1100. doi:10.1111/jep.12069

Bustinza, R., Lebel, G., Gosselin, P., Belanger, D., \& Chebana, F. (2013). Health impacts of the July 2010 heat wave in 
Quebec, Canada. BMC Public Health, 13(56).

doi:10.1186/1471-2458-13-56

Charbonneau, J., Ouellette, F.-R., Gaudet, S., Lecomte, Y., Lesage, A., \& Lefebvre, Y. (2000). Les impacts psychosociaux de la tempête de verglas au Québec. Santé Mentale $A u$ Québec, 25(1), 138-162. doi:10.7202/013028ar

Climat Québec. (2015). Suivi du climat - Bilans d'évènements météo. Repéré à http://www.climat-quebec.qc.ca/ home.php?id=summary_weather_events\&mpn =climate_mon

Communauté Métropolitaine de Montréal. (2015). Projet ILEAU. Repéré à http://cmm.qc.ca/evenements/agora2015/projets-inspirants/liste-de-projets-inspirants/fiche-de -projet/?inspid $=22$

Côté, H., Logan, T., \& Paquin, D. (2015). L’urgence d'agir : L'évaluation climatique au Québec. Vie économique, 6(2), 110. Répéré à http://www.eve.coop/?a=226

Doucet, L., \& Favreau, L. (1991). Théorie et pratiques en organisation communautaire. Service social, 4O(3), 164167.

Doyon, B., Bélanger, D., \& Gosselin, P. (2008). The potential impact of climate change on annual and seasonal mortality for three cities in Québec, Canada. International Journal of Health Geographics, 7(23). doi:10.1186/1476-072X-7 $-23$

Edwards, N. (1999). Population health: Determinants and interventions. Revue canadienne de santé publique, 9o(1), 10-11. doi:10.17269/cjph.90.1173

Edwards, N., \& Di Ruggiero, E. (2011). Exploring which context matters in the study of health inequities and their mitigation. Scandinavian Journal of Public Health, 396 Suppl.), 43-49. doi:10.1177/1403494810393558

Edwards, N., MacLean, L., Estable, A., \& Meyer, M. (2006). Multiple interventions program recommendation for MHPSG technical review. Repéré à http://aix1.uottawa.ca/ nedwards/chru/english/word/MIPS\%20MHPSG\% 20RECOMM\%20\%20March\%2031.doc

Edwards, N., Mill, J., \& Kothari, A. R. (2004). Multiple intervention research programs in community health. Revue canadienne de recherche en sciences infirmières, 36(1), 4054 .

Environnement Canada. (2013a). Le Saint-Laurent et le réchauffement climatique. Repéré à https://www.ec.gc.ca/ stl/default.asp?lang=Fr\&n=4BFoEFoC -1
Environnement Canada. (2013b). Évaluation scientifique canadienne du smog faits saillants et messages clés. Repéré à https://www.ec.gc.ca/Publications/ADo24B6B-A18B408D-ACA259B1B4E04863\%5CEvaluationScientifique CanadienneDuSmogFaitsSaillantsEtMessagesCles.pdf

Evans, R. G., \& Stoddart, G. L. (1990). Producing health, consuming health care. Social Science \& Medicine, 31(12), 1347-1363. doi:10.1016/0277-9536(90)90074-3

Finkelstein, M. M., Jerrett, M., DeLuca, P., Finkelstein, N., Verma, D. K., Chapman, K., \& Sears, M. R. (2003). Relation between income, air pollution and mortality: A cohort study. Canadian Medical Association Journal, 169(5), 397402.

Fondation David Suzuki. (2014). Les changements climatiques au Québec. Repéré à http://www.davidsuzuki.org/fr/ champs-dintervention/changements-climatiques/enjeux-et -recherche/le-canada-et-les-changements-climatiques/leschangements-climatiques-au-quebec/

Giguère, M. (2009). Mesures de lutte aux îlots de chaleur urbains. Repéré à https://www.inspq.qc.ca/pdf/ publications/988_MesuresIlotsChaleur.pdf

Golden, S. (2013). Urban agriculture impacts: Social, health, and economic: A literature review. Repéré à http:// asi.ucdavis.edu/programs/sarep/publications/food-andsociety/ualitreview-2013.pdf

Gower, S. (2011). Protecting vulnerable people from health impacts of extreme heat. Toronto, Ont. Repéré à http:// www.climateontario.ca/doc/ORAC_Products/TPH/ Protecting\%20Vulnerable\%2oPeople\%2ofrom\%2oHealth\% 2oImpacts\%20of\%2oExtreme\%2oHeat.pdf

Greaves, L., \& Bialystok, L. (2011). Health in all policies All talk and little action? Canadian Journal of Public Health, 102(6), 407-409. doi:10.17269/cjph.102.2691

Groupe d'experts intergouvernemental sur les changements climatiques. (2014). Climate change 2014 : Impacts, adaptation and vulnerability. Repéré à https://www.ipcc.ch/ pdf/assessment-report/ar5/wg2/WGIIAR5-

FrontMatterA_FINAL.pdf

Haines, A., McMichael, A. J., \& Epstein, P. R. (2000). Environment and Health: 2. Global Climate Change and Health. Canadian Medical Association. Journal, 163(6), 729-734.

Hancock, T. (2009). Act locally: Community-based population health promotion. A report for the senate subcommittee on population health. Repéré à http:// 
Revue interdisciplinaire des sciences de la santé | Interdisciplinary Journal of Health Sciences

www.parl.gc.ca/content/sen/committee/402/popu/rep/ appendixbjuno9-e.pdf

Health Resources in Action. (2013). Defining healthy communities. Repéré à http://www.hria.org/uploads/ catalogerfiles/defining-healthy-communities/ defining_healthy_communities_1113_final_report.pdf

Heinze, J. (2011). Benefits of green space: Recent research. Repéré à http://www.ehrf.info/wp-content/ uploads/2011/o9/BenefitsofGreenSpace.pdf

Hoffman, S. (2009). Preparing for disaster: Protecting the most vulnerable in emergencies. UC Davis Law Review, 42 (5), 1493-1546.

Institut national de santé publique du Québec. (2010a). Changements climatiques : Qu'est-ce qu'on entend par changements climatiques? Repéré à http:// www.monclimatmasante.qc.ca/changementsclimatiques.aspx

Institut national de santé publique du Québec. (2010b). Ilots de chaleur : Mon climat, ma santé. Repéré à http:// www.monclimatmasante.qc.ca/public/\%C3\%AElots-dechaleur.aspx

Institut national de santé publique du Québec. (2010c). Les changement climatique accentuent les risques d'insécurité alimentaire. Repéré à http://www.monclimatmasante.qc. ca/public/ins\%C $3 \%$ A9curite-alimentaire.aspx

Institut national de santé publique du Québec. (2014). Inondations : État de situation des responsabilités et pratiques en santé environnementale. Repéré à https:// www.inspq.qc.ca/pdf/publications/1955_Inondations_ Etat_Situation_Responsabilites_Pratiques.pdf

Institut national de santé publique du Québec. (2015). Le Projet ILEAU : Pour lutter contre les îlots de chaleur! Repéré à http://www.monclimatmasante.qc.ca/le-projet-ileau -pour-lutter-contre-les-îlots-de-chaleur.aspx

Institut de la statistique du Québec. (2015). Le Québec chiffres en main, édition 2015. Repéré à http://www.stat. gouv.qc.ca/quebec-chiffre-main/pdf/qcm2015_fr.pdf

Institute of Medicine. (2015). Healthy, resilient, and sustainable communities after disasters: Strategies, opportunities, and planning for recovery. Repéré à http:// nationalacademies.org/hmd/ /media/Files/Report\% 20Files/2015/Disaster/postdisaster\%20RB\%20FINAL.pdf

Interventions locales en environnement et aménagement urbain. (2015). Présentation du projet ILEAU. Repéré à http://ileau.ca/presentation-projet-ileau

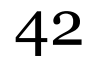

Keller, R. C. (2013). Place matters: Mortality, space, and urban form in the 2003 Paris heat wave disaster. French Historical Studies, 36(2), 299-330. doi:10.1215/001610711960682

Jabareen, Y. (2013). Planning the resilient city: Concepts and strategies for coping with climate change and environmental risk. Cities, 31, 220-9. doi:10.1016/j.cities.2012.

05.004

Klinenberg, E. (1999). Denaturalizing disaster: A social autopsy of the 1995 Chicago heat wave. Theory and Society, 28(2), 239-295. doi:10.1023/A:1006995507723

Krieger, N. (2012). Methods for the scientific study of discrimination and health: An ecosocial approach. American Journal of Public Health, 102(5), 936-944. doi:10.2105/ AJPH.2011.300544

Laaidi, K. (2012). Rôle des îlots de chaleur urbains dans la surmortalité observée pendant les vagues de chaleur-Synthèse des études réalisées par l'Institut de veille sanitaire sur la vague de chaleur d'août 2003. Repéré à http:// www.invs.sante.fr/Publications-et-outils/Rapports-etsyntheses/Environnement-et-sante/2012/Role-des-îlots-de -chaleur-urbains-dans-la-surmortalite-observee-pendantles-vagues-de-chaleur

Last, J. (2010). Future challenges to health and public health services in Canada. Canadian Journal of Public Health, 101(1), 5-19.

Lewis, K., \& Witham, C. (2012). Agricultural commodities and climate change. Climate Policy, 12(Suppl. 1), 53-61.

Litman, T. (2015). Evaluating complete streets: The value of designing roads for diverse modes, users and activities. Repéré à http://www.vtpi.org/compstr.pdf

Maltais, D., \& Gauthier, S. (2008). Les catastrophes dites naturelles : Un construit social ? Présenté à la 4e Conférence canadienne sur les géorisques : Des causes à la gestion, Québec. Repéré à http://www.landslides.ggl.ulaval.ca/ geohazard/o_Keynotes/maltais.pdf

Ministère du développement durable, de l'environnement et de la lutte contre les changements climatiques. (2015). Les gaz à effet de serre. Repéré à http:// www.mddelcc.gouv.qc.ca/air/questce-ges.htm

Navarro, V. (2002). The political economy of social inequalities: Consequences for health and quality of life. New York, NY: Baywood Pub Co.

Neidell, M. J. (2004). Air pollution, health, and socioeconomic status: The effect of outdoor air quality on child- 
Revue interdisciplinaire des sciences de la santé | Interdisciplinary Journal of Health Sciences

hood asthma. Journal of Health Economics, 23(6), 12091236. doi:10.1016/j.jhealeco.2004.05.002

New York City Department of Transportation. (2012). Measuring the street: New metrics for 21st century streets. Repéré à http://www.nyc.gov/html/dot/downloads/pdf/201210-measuring-the-street.pdf

Patrick, R., Capetola, T., Townsend, M., \& Nuttman, S. (2012). Health promotion and climate change: Exploring the core competencies required for action. Health Promotion International, 27(4), 475-485. doi:10.1093/heapro/ daro55

Patrick, R., Noy, S., \& Henderson-Wilson, C. (2015). Urbanisation, climate change and health equity: How can health promotion contribute? International Journal of Health Promotion and Education, 54(1), 1-16.

doi:10.1080/14635240.2015.1057653

Pegg, S. (2011). Bilan-faim 2011 : Rapport sur la faim et l'utilisation des banques alimentaires au Canada, qui propose des recommandations pour le changement. Repéré à https://www.foodbankscanada.ca/getmedia/469b34d976b6-4d14-b55d-ee9117837903/Bilan-Faim2012.pdf.aspx

Santé Canada. (2012). Canadiens en santé : Chaleur accablante-vagues de chaleur. Repéré à http://canadiensen sante.gc.ca/healthy-living-vie-saine/environmentenvironnement/sun-soleil/heat-extreme-chaleur-fra.php

Seskin, S., \& Gordon-Koven, L. (2013). The best complete streets policies of 2012. Repéré à http://www.smartgrowth america.org/complete-streets-2012-analysis

Sheffield, P. E., \& Landrigan, P. J. (2011). Global climate change and children's health: Threats and strategies for prevention. Environmental Health Perspectives, 119(3), 291298. doi:10.1289/ehp.1002233

Smart Growth America. (2015). Implementation complete streets policy. Repéré à http://www.smartgrowthamerica. org/complete-streets/implementation

Smith, K. R., Woodward, A., Campbell-Lendrum, D., Chadee, D. D., Honda, Y., Qiyong, L.,...Yamamoto, S. (2014). Human health: Impacts, adaptation, and cobenefits. In: Climate Change 2014: Impacts, Adaptation, and Vulnerability Part A: Global and Sectoral Aspects Con tribution of Working Group II to the Fifth Assessment Report of the Intergovernmental Panel of Climate Change. Cambridge, UK: Cambridge University Press.

Suarez-Balcazar, Y., Martinez, L. I., Jayraj, A., \& Cox, G. (2006). African Americans' views on access to healthy foods: What a farmers' market provides. Journal of Extension, 44(2), 31-43.

Toronto Centre for Active Transportation. (2015). Complete streets for Canada: Policy and design hub for Building safe and inviting streets for all. Repéré à http://complete streetsforcanada.ca/

Transport Canada. (2009). Complete streets: Making Canada's roads safer for all Ottawa: Case studies in sustainable transportation. Repéré à http://publications. gc.ca/ collections/collection_2012/tc/T41-1-72-eng.pdf

Union des municipalités du Québec. (2008). Politique de mobilité et transport durables. Repéré à http:// old.umq.qc.ca/uploads/files/pub_autres/enjeux_defis.pdf

Victorian Council of Social Service. (2014, Juin 13). Disaster and disadvantage: Social vulnerabilty in emergency management. Retrieved from http://vcoss.org.au/ documents/2014/06/VCOSS_Disadvantage-anddisaster_2014.pdf

Walpole, S. C., Rasanathan, K., \& Campbell-Lendrum, D. (2009). Natural and unnatural synergies: Climate change policy and health equity. Bulletin of the World Health Organization, 87(10), 799-801. doi:10.1590/Soo42-96862 009001000017

Warren, F. J., \& Lemmen, D. S. (2014). Vivre avec les changements climatiques au Canada: Perspectives des secteurs relatives aux impacts et à l'adaptation. Repéré à https:// www.rncan.gc.ca/sites/www.nrcan.gc.ca/files/earthscien ces/pdf/assess/2014/pdf/Rapport-complet_Fra.pdf

Watts, N., Adger, W. N., Agnolucci, P., Blackstock, J., Byass, P., Cai, W.,...Costello, A. (2015). Health and climate change: Policy responses to protect public health. The Lancet, 386 (10006), 1861-1914.

Wilkinson, R. G. (2010). L'égalité c'est la santé. Paris, France : Demopolis.

Wong, C.-M., Ou, C.-Q., Chan, K.-P., Chau, Y.-K., Thach, T.Q., Yang, L.,...Lam, T.-H. (2008). The effects of air pollution on mortality in socially deprived urban areas in Hong Kong, China. Environmental Health Perspectives, 116(9), 11891194.

Zupancic, T., Bulthuis, M., \& Westmacott, C. (2015). The impact of green space on heat and air pollution in urban communities: A meta-narrative systematic review. Repéré à http://www.davidsuzuki.org/publications/

ImpactofGreenSpaceonHeatandAirPollutioninrbanCommunities.pdf 